\title{
A characterization of homomorphisms \\ in certain Banach involution algebras
}

by

GEORGE MALTESE (Münster) and REGINA。WILLE-FIER (München)

Abstract. The following question related to the Gleason-Kahane-Żelazko theorem is discussed: If $\varphi$ is a continuous linear form on a Banach algebra $A$ such that every element in $\operatorname{ker} \varphi$ belongs to the kernel of some nontrivial multiplicative form, does it follow that $\varphi=c h$ for some $c \neq 0$ and some nontrivial multiplicative form $h$ ? An affirmative answer is given for a class of Banach involution algebras. Applications to $C_{0}(X), X$ locally compact, and to $L^{1}(G), G$ a locally compact metrizable group, are given.

0. Introduction. In this paper we discuss the following question related to the Gleason-Kahane-Żelazko theorem: Suppose $\varphi$ is a continuous linear functional on a complex Banach algebra $A$ such that every element in $\operatorname{ker} \varphi$ is in the kernel of some complex homomorphism $(\neq 0)$ on $A$. Does it follow that $\varphi=c h$ for some $0 \neq c \in C$ and some nontrivial complex homomorphism $h$ on $A$ ? It is shown that this question has an affirmative answer if $A$ is a complex involutive Banach algebra such that the space $\Delta_{A}$ of nontrivial complex continuous homomorphisms of $A$ endowed with the relative $\sigma\left(A^{\prime}, A\right)$ topology is sigma-compact and the involution is symmetric, i.e. $h(x)$ is real for every selfadjoint $x$ and every $h$ in $\Delta_{A}$. On the other hand, the hypothesis of sigma-compactness of $\Delta_{A}$ turns out to be an essential assumption since it is necessary in order to guarantee the existence of at least one element in $A$ which belongs to no regular maximal ideal of codimension 1.

In the sequel we consider two important examples. First, the above characterization always holds for the convolution algebra $L^{1}(G)$ of a metrizable group $G$. Moreover, in the case of an abelian group $G$ the essential hypothesis of sigma-compactness of the space $\Delta_{L^{1}(G)}$ is fulfilled if and only if $L^{1}(G)$ possesses a sequential approximate identity. Similarly for the Banach algebra $C_{0}(X)$ of continuous complex-valued functions vanishing at infinity on a locally compact Hausdorff space $X$, the sigma-compactness of $X$ (and

Mathematics Subject Classification: Primary 46H10; Secondary 46K05.

Key words and phrases: Banach algebra, homomorphism, Gleason-Kahane-Żelazko theörem 
consequently the sigma-compactness of $\left.\Delta_{C_{n}(X)}\right)$ is equivalent to the existence of a sequential approximate identity in the algebra $C_{0}(X)$.

1. Preliminaries and basic assumptions. Throughout this paper "algebra" means "complex associative algebra" and "homomorphism" means "complex homomorphism". In a commutative Banach algebra $A$ with identity the famous theorem of Gleason-Kahane-Żelazko gives the following characterization of maximal ideals (see [2], [4] and [13]): Let $m$ be a subspace of codimension 1 in $A$. Then $m$ is a maximal ideal if and only if each element of $m$ belongs to some maximal ideal.

It is natural to ask whether this characterization holds for the regular maximal ideals of a commutative Banach algebra without unit element. In 1969 Warner and Whitley considered this problem in [10] and they showed that there are commutative Banach algebras in which every element belongs to some regular maximal ideal. Hence apparently the above characterization cannot be valid for arbitrary Banach algebras without identity.

On the other hand, Warner and Whitley did obtain the above characterization in two important cases: first, for the convolution algebra $L^{\mathbf{1}}(G)$ of a metrizable abelian topological group $G$, and secondly, for a Banach algebra generated by one element. In the second case they need the additional hypothesis that the considered subspace be closed.

Throughout this paper we prefer to work with multiplicative linear functionals rather than with regular maximal ideals. Moreover, we shall use some notation, which is familiar for commutative Banach algebras, also in the noncommutative case: so $\Delta_{A}$ will denote the set of all nontrivial homomorphisms on a Banach algebra $A$. In the case of a commutative Banach algebra it is well known from Gelfand theory that $\Delta_{A}$ may be identified with the set of regular maximal ideals, since any regular maximal ideal has codimension 1. For a noncommutative Banach algebra $A$ the codimension of a regular maximal ideal in $A$ is not necessarily 1 ; hence, in general, $\Delta_{A}$ must be identified with the set of regular maximal ideals with codimension 1 (see $19 \mathrm{C}$ in [15]).

In general, the existence of nontrivial continuous homomorphisms, especially for a noncommutative Banach algebra without unit element, is not guaranteed. Throughout this paper we shall assume that the space $\Delta_{A}$ of the considered Banach algebra is not empty. In fact, this hypothesis is fulfilled for the two important examples which are discussed in the last two sections.

For any element $x$ in a Banach algebra $A$ the Gelfand transform of $x$ is defined by $\hat{x}: \Delta_{A} \rightarrow C, h \mapsto h(x)$. If $\Delta_{A}$ is endowed with the Gelfand topology (i.e. the weakest topology on $\Delta_{A}$ for which every $\hat{x}$ is continuous), then $\Delta_{A}$ is a locally compact Hausdorff space such that every Gelfand transform vanishes at infinity (see 19B in [5]).

Thus for this paper the problem of Warner and Whitley [10] is reformulated in the following way: Let $A$ be a Banach algebra and let $\varphi$ be a continuous linear functional on $A$. Is $\varphi$ the scalar multiple $(\neq 0)$ of some homomorphism $(\neq 0)$ on $A$ if it is known that every element in the kernel of $\varphi$ belongs to the kernel of some homomorphism $(\neq 0)$ on $A$ ?

In contrast to $[10]$ we do not suppose that the considered Banach algebra is commutative. Hence the above formulation just characterizes those regular maximal ideals which are of codimension 1 .

In the first section of this paper we obtain the following result (Theorem 2.3) $\left({ }^{1}\right)$ : the above question has an affirmative answer whenever $A$ is an involutive Banach algebra such that the space $\Delta_{A}$ is sigma-compact (i.e. a countable union of compact sets) and the involution in $A$ is symmetric $\left({ }^{2}\right)$.

Moreover, we prove that the hypothesis of the sigma-compactness of $\Delta_{A}$ is fulfilled if the Banach algebra $A$ possesses a sequential approximate identity. In this context we also show that the hypothesis of the sigmacompactness of $\Delta_{A}$ is an essential assumption, since it is necessary in order to guarantee the existence of at least one element in $A$ which belongs to no regular maximal ideal of codimension 1 .

The last two sections of this paper treat two important examples. First we examine the Banach algebra $C_{0}(X)$ of continuous complex-valued functions vanishing at infinity on a locally compact Hausdorff space $X$. It is shown that the above characterization of homomorphisms holds for $C_{0}(X)$ if and only if the algebra $C_{0}(X)$ possesses a countable approximate identity and that this latter condition is equivalent to the sigma-compactness of $X$.

The second example is the convolution algebra $L^{1}(G)$ of a locally compact topological group $G$. The above characterization of homomorphisms is realized even for a nonabelian metrizable group. In the case of an abelian group $G$ the characterization holds if and only if $G$ is metrizable. In analogy to the algebra $C_{0}(X)$, the characterization for commutative convolution algebras is equivalent to the existence of a sequential approximate identity in $L^{1}(G)$

We wish to thank Gerd Niestegge for useful discussions during the preparation of this paper. Special thanks are due to the referee for many constructive comments which simplified the exposition.

2. A generalization of the Theorem of Gleason-Kahane-Zielazko. If the above characterization of homomorphisms is valid for a Banach algebra $A$ and, on the other hand, if there exist linear functionals $(\neq 0)$ on $A$ which are scalar multiples of no homomorphism on $A$, then there is an element $w$ in $A$

(1) Recently our attention was drawn to the fact that a commutative version of this theorem was proved by Warner and Whitley in [11]. Our noncommutative proof relies on different methods.

$\left.{ }^{2}\right)$ The involution is symmetric if and only if $h\left(x^{*}\right)=\overline{h(x)}$ for each $h$ in $\Delta_{A}$ and $x$ in $A$. 
with the property $h(w) \neq 0$ for all $h$ in $\Delta_{A}$. In particular, in the case of a Banach algebra with unit element $e$ the unit element never belongs to the kernel of a homomorphism $(\neq 0)$ on $A$. Hence the hypothesis that an element $w$ exists for which $h(w) \neq 0$ for all $h$ in $\Delta_{A}$ weakens in a natural way the condition that the Banach algebra $A$ be unital.

The following theorem shows that this weakening is also expressed in the topological properties of the space $\Delta_{A}$ : the compact maximal ideal space of a commutative unital algebra is replaced by the sigma-compact space $\Delta_{A}$ in the general case.

TheOREM 2.1. Let $A$ be a complex Banach algebra and let $w$ be an element in $A$ such that $h(w) \neq 0$ for all $h$ in $\Delta_{A}$. Then the space $\Delta_{A}$ is sigma-compact in the Gelfand topology. Furthermore, if $A$ has a symmetric involution, then the converse implication also holds; viz. if $\Delta_{A}$ is sigma-compact, then there exists $w \in A$ with $h(w) \neq 0$ for all $h$ in $\Delta_{A}$.

Proof. The first assertion follows by writing

$$
\Delta_{A}=\bigcup_{n=1}^{\infty}\left\{h \in \Delta_{A}:|h(w)| \geqslant 1 / n\right\}
$$

since every term in this countable union is compact ( $\hat{w}$ vanishes at infinity). The second assertion follows from the fact that for every $h$ in $\Delta_{A}$ there exists $x_{h}$ in $A$ with $h\left(x_{h}\right) \neq 0$. Since $A$ has a symmetric involution we have $h\left(x^{*} x\right)$ $\geqslant 0$ for all $h$ in $\Delta_{A}$ and all $x$ in $A$. The relation $h\left(x_{h}^{*} x_{h}\right)>0$ implies that there is a neighborhood $V_{h}$ of $h$ in $\Delta_{A}$ with $\varphi\left(x_{h}^{*} x_{h}\right)>0$ for all $\varphi$ in $V_{h}$. We have $\Delta_{A}=\bigcup\left\{V_{h}: h \in \Delta_{A}\right\}$ and since $\Delta_{A}$ is sigma-compact, we can choose from $\left\{V_{h}\right\}_{h \in A_{A}}$ a countable subcover $\left\{V_{h_{k}}\right\}_{k=1}^{\infty}$. For each $k^{*}$ let $u_{k}:=x_{h_{k}}^{*} x_{h_{k}}$ and finally let

$$
w:=\sum_{k=1}^{\infty} \frac{u_{k}}{2^{k}\left\|u_{k}\right\|}
$$

Clearly $h(w)>0$ for all $h$ in $\Delta_{A}$ and the conclusion follows.

That the converse implication in Theorem 2.1 may fail if the involutive Banach algebra is not symmetric is shown in the following example (which is also discussed in [10]):

EXAMPLE. Let $A$ be the uniform algebra of all functions of two complex variables which are continuous on the bidisc $\Delta^{2}:=\left\{(\lambda, \mu) \in C^{2}:|\lambda| \leqslant 1\right.$, $|\mu| \leqslant 1\}$ and holomorphic in its interior. Let $M_{0}$ be the maximal ideal of $A$ defined by $M_{0}:=\{f \in A: f(0,0)=0\}$. Then $M_{0}$ is a Banach algebra with a nonsymmetric involution given by $f^{*}(\lambda, \mu):=\overline{f(\bar{\lambda}, \bar{\mu})}$. Clearly we have $\Delta_{M_{0}}$ $=\Delta^{2} \backslash\{(0,0)\}$ and so $\Delta_{M_{0}}$ is sigma-compact. On the other hand, $M_{0}$ does not contain any nonvanishing function since every holomorphic function on
$\Delta^{2}$ in two complex variables which vanishes at $(0,0)$ must vanish also on an analytic subset of $\Delta^{2}$ which meets its boundary.

Theorem 2.1 shows that for the above-discussed version of the theorem of Gleason-Kahane-Żelazko it suffices to consider Banach algebras with $\Delta_{A}$ sigma-compact. On the other hand, this essential hypothesis is always fulfilled if the Banach algebra possesses a sequential approximate identity. For the sake of completeness we include a proof of this result.

THEOREM 2.2. Let $A$ be a Banach algebra with a countable left (resp. right) approximate identity $u_{n}$. Then the maximal ideal space of $A$ is sigma-compact.

Proof. Let $u_{n}$ be a left approximate identity (the proof for a right approximate identity is analogous). Since for any $h$ in $\Delta_{A}$ there is an element $x_{0}$ in $A$ such that $h\left(x_{0}\right) \neq 0$, the relation $h\left(u_{n} x\right) \rightarrow h(x)$ for all $h$ in $\Delta_{A}$ and $x$ in $A$ implies $h\left(u_{n}\right) \rightarrow 1$ for all $h$ in $\Delta_{A}$. It follows that the sets

$$
K_{n}:=\left\{h \in \Delta_{A}:\left|\hat{u}_{n}(h)\right| \geqslant \frac{1}{2}\right\}, \quad n=1,2, \ldots,
$$

form the desired countable covering of $\Delta_{A}$ : for since the Gelfand transforms $\hat{u}_{n}$ vanish at infinity, every $K_{n}$ is compact in $\Delta_{A}$. Furthermore, since $h\left(u_{n}\right) \rightarrow 1$ for all $h$ in $\Delta_{A}$, each $h$ belongs to some $K_{n}$.

The following theorem solves the characterization problem formulated in the introduction for every involutive symmetric Banach algebra $A$.

THEOREM 2.3. Let $A$ be a Banach algebra with $\Delta_{A}$ sigma-compact and with a symmetric involution. Then for every linear functional $\varphi$ the following conditions are equivalent:

(i) There is a $\lambda \in C \backslash\{0\}$ such that $\lambda \varphi$ is in $\Delta_{A}$.

(ii) For every $x$ in the kernel of $\varphi$ there is a homomorphism $h$ in $\Delta_{A}$ such that $x$ belongs to the kernel of $h$.

Proof. Since any homomorphism on $A$ has property (ii), the implication (i) $\Rightarrow$ (ii) is obvious.

In order to prove the converse implication let $\varphi$ be a linear functional on $A$ such that (ii) holds. According to Theorem 2.1 there is an element $w$ in $A$ with $h(w) \neq 0$ for every $h$ in $\Delta_{A}$. Considering $w w^{*}$ we may suppose $w$ to be selfadjoint. Now property (ii) implies $\varphi(w) \neq 0$ and $\varphi\left(w^{2}\right) \neq 0$. Since the linear functional $\lambda \varphi$ fulfills (ii) for any $\lambda \in C \backslash\{0\}$, we may even assume that $\varphi(w)=1$.

Hence without loss of generality we may reduce to the following situation: $\varphi$ is a linear functional on $A$ with property (ii), $\varphi(w)=1$ and $\varphi\left(w^{2}\right) \neq 0$ for a selfadjoint $w$ such that $h(w) \neq 0$ for all $h$ in $\Delta_{A}$.

We shall show that the mapping $F: A \rightarrow C$ defined by

$$
F(x):=\varphi\left(w^{2}\right) \varphi(x) \quad(x \in A)
$$


is a homomorphism. We have $F(w)=\varphi\left(w^{2}\right)$ and $F\left(w^{2}\right)=\varphi\left(w^{2}\right) \varphi\left(w^{2}\right)$ so that

$$
F\left(\dot{w}^{2}\right)=[F(w)]^{2} .
$$

By definition $\operatorname{ker} F=\operatorname{ker} \varphi$ and so by (ii), $F(x)=0$ implies $h(x)=0$ for some $h \in \Delta_{A}$ ( $h$ depends on $x$ ).

By (1), $F\left(w^{2}-F(w) w\right)=0$ so there exists $h$ in $\Delta_{A}$ with $h\left(w^{2}\right)$ $=h(w) F(w)$; hence $F(w)$ is real. For any selfadjoint $u$ we have

$$
F\left(u-\frac{w}{F(w)} F(u)\right)=0
$$

so there exists $g$ in $\Delta_{A}$ with $g(u)=(g(w) / F(w)) F(u)$, and so $F(u)$ is real for each selfadjoint $u$. For any $x, y$ in $A$ we have

$$
F\left(x y-y x-\frac{w}{F(w)} F(x y-y x)\right)=0
$$

so there exists $k$ in $\Delta_{A}$ with $k(x y-y x)=(k(w) / F(w)) F(x y-y x)$, and so we obtain

$$
F(x y)=F(y x) \quad \text { for all } x, y \in A .
$$

For any selfadjoint $u$ we have

$$
F\left(u-\frac{w}{F(w)} F(u)+i\left\{u^{2}-\frac{w^{2}}{F\left(w^{2}\right)} F\left(u^{2}\right)\right\}\right)=0
$$

and hence there exists $l$ in $\Delta_{A}$ with

$$
l\left(u-\frac{w}{F(w)} F(u)+i\left\{u^{2}-\frac{w^{2}}{F\left(w^{2}\right)} F\left(u^{2}\right)\right\}\right)=0 .
$$

Since $F(u), F\left(u^{2}\right), F(w), F\left(w^{2}\right)$ are real, the elements $u-(w / F(w)) F(u)$ and $u^{2}$ $-\left(w^{2} / F\left(w^{2}\right)\right) F\left(u^{2}\right)$ are selfadjoint and so since the involution is symmetric, (3) implies

$$
l(u)=\frac{l(w)}{F(w)} F(u), \quad l\left(u^{2}\right)=\frac{l\left(w^{2}\right)}{F\left(w^{2}\right)} F\left(u^{2}\right) .
$$

Together with (1) this gives

$$
F\left(u^{2}\right)=\frac{l\left(u^{2}\right)}{l\left(w^{2}\right)} F\left(w^{2}\right)=\left[\frac{l(u)}{l(w)} F(w)\right]^{2}=[F(u)]^{2} .
$$

The relations (2) and (4) show that for any selfadjoint $u, v$ in $A$

$$
\begin{aligned}
2 F(u v) & =F(u v+v u)=F\left((u+v)^{2}-u^{2}-v^{2}\right) \\
& =(F(u)+F(v))^{2}-F\left(u^{2}\right)-F\left(v^{2}\right)=2 F(u) F(v) .
\end{aligned}
$$

This immediately implies that $F(x y)=F(x) F(y)$ for arbitrary $x, y$ in $A$ and completes the proof.

Remark. The above proof represents a generalization of the proof of Corollary 1.1 in [13] for a Banach algebra without unit element.

\section{Characterization of homomorphisms on $C_{0}(X)$ in the sense of Theorem} 2.3. In this section we consider the commutative Banach algebra $C_{0}(X)$ of continuous complex-valued functions vanishing at infinity on a locally compact Hausdorff space $X$. If $X$ is not sigma-compact, then the maximal ideal space of $C_{0}(X)$ is not sigma-compact either; hence Theorem 2.1 implies that any element of $C_{0}(X)$ belongs to a regular maximal ideal of $C_{0}(X)$. But generally there will be linear functionals on $C_{0}(X)$ which are scalar multiples of no homomorphism on $C_{0}(X)$. Hence in the case of a non-sigma-compact space $X$ the homomorphisms on $C_{0}(X)$ will hardly be characterized by property (ii) of Theorem 2.3 .

If on the other hand the space $X$ is sigma-compact, then the homomorphisms on $C_{0}(X)$ will always be characterized by condition (ii) of Theorem 2.3:

THEOREM 3.1. Let $X$ be a locally compact Hausdorff space and let $F$ be a linear functional $(\neq 0)$ on $C_{0}(X)$. Then $F$ is representable in the form

$$
F(f)=\lambda f\left(x_{0}\right) \quad\left(f \in C_{0}(X)\right)
$$

for some $x_{0} \in X$ and some $\lambda \in C \backslash\{0\}$ if and only if every function in the kernel of $F$ has a zero in $X$.

Proof. The assertion is a direct consequence of Theorem 2.3 if one uses the well-known fact that any homomorphism on $C_{0}(X)$ is the point evaluation at some point in $X$.

Thus the characterization of homomorphisms on $C_{0}(X)$ by property (ii) of Theorem 2.3 is possible if and only if the space $X$ is sigma-compact. Another necessary and sufficient condition is that $C_{0}(X)$ possesses an approximate identity sequence.

THEOREM 3.2. Let $X$ be a locally compact Hausdorff space. Then there is an approximate identity of $C_{0}(X)$ which is a sequence if and only if $X$ is sigmacompact.

Proof. If $C_{0}(X)$ possesses a countable approximate identity, then Theorem 2.3 implies that $\Delta_{C_{0}(X)}$ is sigma-compact; hence $X$ is sigma-compact too.

For the converse implication let $X$ be the countable union of compact sets $K_{i}$. Since $X$ is locally compact, every $K_{i}$ is covered by a finite number of open sets $U_{i, k}$ with compact closure in $X$. Moreover, for every $i$ the union of 
the $\bar{U}_{i, k}$ is a compact set containing $K_{i}$ in its interior. Hence without loss of generality we may choose compact sets $K_{i}$ such that

$$
X=\bigcup_{i=1}^{\infty} K_{i}
$$

(here $\check{K}_{i}$ denotes the interior of $K_{i}$ ). Considering the sets

$$
C_{n}:=\bigcup_{i=1}^{n} K_{i}
$$

we may finally choose a sequence of compact sets $C_{n}$ with

$$
\bigcup_{n=1}^{\infty} \stackrel{C}{C}_{n}=X \quad \text { and } \quad C_{n+1} \supset C_{n} \text { for every } n \in N \text {. }
$$

Now the countable approximate identity is defined in the following way: for every $C_{n}$ the Lemma of Urysohn (see Proposition 7.1.8 in [1]) guarantees the existence of a function $u_{n}$ in $C_{0}(X)$ such that $u_{n}$ is real-valued with $0 \leqslant u_{n} \leqslant 1$ and $u_{n}(x)=1$ for every $x \in C_{n}$.

In fact, it is easy to show that $u_{n}$ is approximate identity: Let $g$ be any element of $C_{0}(X)$ and let $\varepsilon>0$. Since $g$ vanishes at infinity, there is a compact set $K_{\varepsilon}$ with $|g(x)|<\varepsilon$ for every $x \notin K_{\varepsilon}$. Now $K_{\varepsilon}$ is contained in the union of the open sets $\dot{C}_{n}$. Hence a finite number of them already cover $K_{\varepsilon}$. Since $C_{n} \subset C_{n+1}$ for all $n$, there is in particular an $m \in N$ such that $K_{\varepsilon} \subset C_{n}$ $\forall n \geqslant m$. Consequently, we have for every $n \geqslant m$

$$
\begin{aligned}
& \left|g u_{n}(t)-g(t)\right|=|g(t)-g(t)|=0 \quad \forall t \in K_{\varepsilon}, \\
& \left|g u_{n}(t)-g(t)\right|=|g(t)|\left|u_{n}(t)-1\right|<\varepsilon \quad \forall t \notin K_{\varepsilon} .
\end{aligned}
$$

Therefore we conclude that $\left\|g u_{n}-g\right\|_{\infty}<\varepsilon$. Thus $g u_{n}$ converges uniformly to $g$, which means that $u_{n}$ is an approximate identity.

4. Characterization of homomorphisms on $L^{1}(G)$ in the sense of Theorem 2.3. Here we consider the convolution algebra $L^{1}(G)$ of a locally compact topological group $G$. Since the space $\Delta_{L^{1}(G)}$ may be identified with the character group $G^{\prime}$ of $G$ (see Corollary 23.7 in [3]), there are always homomorphisms on $L^{1}(G)$.

The following theorem shows that the characterization of homomorphisms on $L^{1}(G)$ in the sense of Theorem 2.3 holds whenever $G$ is metrizable (even in the noncommutative case!):

THEOREM 4.1. Let $G$ be a metrizable locally compact topological group. Let $\varphi$ be a linear functional on $L^{1}(G)$ with the following property (here $\lambda$ denotes a left Haar measure): If $\varphi(f)=0$, there exists $x_{f}^{\prime} \in G^{\prime}$ with

$$
\int_{G} f(x) \overline{x_{f}^{\prime}(x)} d \lambda(x)=0 .
$$

Then there are $\mu \in C \backslash\{0\}$ and a character $x^{\prime}$ such that

$$
\varphi(f)=\mu \int_{G} f(x) \overline{x^{\prime}(x)} d \lambda(x) \quad \forall f \in L^{1}(G) .
$$

Proof. We have to verify the hýpothesis of Theorem 2.3. According to Satz 5.8 in [6], $L^{1}(G)$ always possesses an approximate identity $e_{U}$. Moreover, the index set of $e_{U}$ is a local base at $e$ in $G$. But if $G$ is metrizable there is a countable local base at $e$ (see Satz 1.1 .13 in [6]); hence $L^{1}(G)$ even possesses an approximate identity which is a sequence. Now Theorem 2.2 implies that $\Delta_{L^{1}(G)}$ is sigma-compact.

We still have to prove that the involution in $L^{1}(G)$ fulfills the symmetry property demanded in Theorem 2.3. For this it suffices to prove that

$$
h\left(f^{*}\right)=\overline{h(f)} \text { for every } h \in \Delta_{L^{1}(G)} \text { and every } f \in L^{1}(G) .
$$

So let $h$ be any homomorphism on $L^{1}(G)$. According to Corollary 23.7 in [3] there is a character $x_{h}^{\prime}$ of $G$ such that

$$
h(f)=\int_{G} f(x) \overline{x_{h}^{\prime}(x)} d \lambda(x) \quad\left(f \in L^{1}(G)\right) .
$$

Hence we have for every $f$ in $L^{1}(G)$

$$
h\left(f^{*}\right)=\int_{G} f^{*}(x) \overline{x_{h}^{\prime}(x)} d \lambda(x)=\int_{G} \Delta\left(x^{-1}\right) \overline{f\left(x^{-1}\right)} x_{h}^{\prime}\left(x^{-1}\right) d \lambda(x) .
$$

Here $\Delta$ denotes the modular function of $G$. Using Theorem 30.B in [5] we conclude that

$$
h\left(f^{*}\right)=\int_{G} \overline{f(x)} x_{h}^{\prime}(x) d \lambda(x)=\overline{\int_{G} f(x) \overline{x_{h}^{\prime}(x)} d \lambda(x)}=\overline{h(f)} .
$$

Thus all the hypotheses of Theorem 2.3 are fulfilled and the assertion follows.

Remark. The proof of Theorem 4.1 implies that the involution in $L^{1}(G)$ always has the symmetry property

$$
h\left(f^{*}\right)=\overline{h(f)} \quad\left(f \in L^{1}(G), h \in \Delta_{L^{1}(G)}\right)
$$

On the contrary, an example in [8] (p. 324) shows that the involution of a noncommutative convolution algebra need not be hermitian (which means that every selfadjoint element in $L^{1}(G)$ has a real spectrum). 
Since for a noncommutative Banach algebra the image of the Gelfand transform $\hat{x}$ is just a subset of the spectrum of $x$, the symmetry property in Theorem 2.3 is of course weaker than the assumption of a hermitian involution.

The last theorem shows that in the case of an abelian group $G$, the hypothesis of metrizability is also necessary in order to characterize homomorphisms on $L^{1}(G)$ by condition (ii) of Theorem 2.3:

THEOREM 4.2. Let $G$ be an abelian topological group. Then the following conditions are equivalent:

(i) $G$ is metrizable.

(ii) There is a countable local base. at $e \in G$.

(iii) $L^{1}(G)$ possesses an approximate identity sequence.

(iv) $\Delta_{L^{1}(G)}$ is sigma-compact.

Proof. As to the equivalence of (i) and (ii) see Satz 1.1.13 in [6] (i) $\Rightarrow$ (iii) is shown in the proof of Theorem 4.1.

According to Theorem 2.2, (iii) $\Rightarrow$ (iv) holds for every Banach algebra. The equivalence of (ii) and (iv) is proved in [3] (see 24.48).

Generally there exist even continuous linear functionals on $L^{1}(G)$ which are scalar multiples of no homomorphism on $L^{1}(G)$ : while the dual space of $L^{1}(G)$ is represented by $L^{\infty}(G)$, the maximal ideal space of $L^{1}(G)$ may be identified with the character group.

Hence for an abelian group $G$ the characterization of homomorphisms on $L^{1}(G)$ by condition (ii) of Theorem 2.3 holds on the one hand if and only if $G$ is metrizable, on the other hand, similarly to $C_{0}(X)$, if and only if $L^{1}(G)$ possesses a countable approximate identity.

\section{References}

[1] D. L. Cohn, Measure Theory, Birkhäuser, Boston 1980.

[2] A. Gleason, A characterization of maximal ideals. J. Analyse Math. 19 (1967), 171-172.

[3] E. Hewitt and K. A. Ross, Abstract Harmonic Analysis, vol. 1, Springer, Berlin 1963.

[4] J.-P. Kahane and W. Żelazko, A characterization of maximal ideals in commutative Banach algebras, Studia Math. 29 (1968), 339-343.

[5] L. H. Loomis, An Introduction to Abstract Harmonic Analysis, van Nostrand, Princeton 1953.

[6] D. Lutz, Topologische Gruppen, B. I.-Wissenschaftsverlag, Zürich 1976.

[7] G. Maltese, Banachalgebren, Vorlesungsskript, Münster 1983.

[8] C. E. Rickart, General Theory of Banach Algebras, van Nostrand, Princeton 1960

[9] W. Rudin, Function Theory in Polydiscs, Benjamin, New York 1969.

[10] C. R. Warner and R. Whitley, A characterization of regular maximal ideals, Pacific J. Math. 30 (1969), 277-281. [11],-- , Ideals of finite codimension in $C[0,1]$ and $L^{1}(R)$, Proc. Amer. Math. Soc. (2) 76
(1979), 263-267.
[12] R. Wille, Korovkin approximation of homomorphisms in involution algebras, Arch. Math. (Basel) 45 (1985), 549-554.

[13] -, The theorem of Gleason-Kahane-Żelazko in a commutative symmetric Banach algebra, Math. Z. 190 (1985), 301-304.

MATHEMATISCHES INSTITUT

UNIVERSITÄT MUNSTER

Einsteinstr. 62, D-4400 Münster, West Germany

and

SIEMENS AG

Hofmannstr. 51, D-8000 München 70, West Germany 